\title{
Muestra Gastronómica de la Tradición Carmelita: Empresa creativa para la difusión de nuevas identidades culturales
}

\section{Gastronomic traditional sample of Ciudad del Carmen: A creative industry for the transmission of new cultural identities}

\author{
Melenie Felipa Guzmán Ocampo ${ }^{1}$ https://orcid.org/0000-0003-4012-3630, \\ José del Carmen Barrientos López ${ }^{1}$ https://orcid.org/0000-0002-8261-0157, María José Guillermo \\ Echeverría 1 https://orcid.org/0000-0001-8829-1997 \\ ${ }^{1}$ Universidad Autónoma del Carmen, Ciudad del Carmen, México \\ mguzman@pampano.unacar.mx, jbarrientosepampano.unacar.mx, \\ mguillermo@pampano.unacar.mx
}

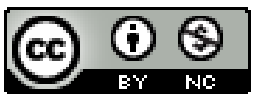

Esta obra está bajo una licencia internacional Creative Commons Atribución-NoComercial 4.0.

$\begin{array}{ll}\text { Enviado: } & 2021 / 11 / 16 \\ \text { Aceptado: } & 2021 / 12 / 06 \\ \text { Publicado: } & 2021 / 12 / 30\end{array}$

\section{Resumen}

La Muestra Gastronómica de la Tradición Carmelita germina de la idea empresarial de un estudiante de Comunicación y Gestión Cultural, como parte de su curso Diseño y Evaluación de Proyectos Emprendedores. El nacimiento de este proyecto tuvo por objetivo dar difusión a la gastronomía de Ciudad del Carmen, Campeche, lugar que por su actividad petrolera recibe a personas de todo el país y el mundo y por ello las identidades cada día se van modificando, afianzado o generando nuevas, el punto de convergencia de la presente investigación es la gastronomía. El proceso metodológico fue la observación empírica de los investigadores de qué alimentos consumían las personas en los lugares públicos como malecones de la ciudad, plazas comerciales, centro histórico, escuelas, mercados, etc., con la finalidad de convocar a los microempresarios de la ciudad a participar en la Primera Muestra Gastronómica Carmelita. En los resultados del proyecto se evidenciaron beneficios económicos que demuestran la sostenibilidad de este, así como la medición de satisfacción de los usuarios de la muestra. Este tipo de empresas creativas permite comprender cómo la identidad alrededor de la gastronomía permite que la diversidad cultural se congregue a degustar un poco de sus culturas mediante el paladar.

Palabras clave: Ciudad del Carmen, Comunicación y Gestión Cultural, Identidad, Gastronomía, Industrias Culturales.

Sumario: Introducción, Metodología, Análisis de resultados, Discusión de resultados y Conclusiones.

Como citar: Guzmán, M., Barrientos, J. C., \& Guillermo, M. J. (2021). Muestra Gastronómica de la Tradición Carmelita: Empresa creativa para la difusión de nuevas identidades culturales. Revista

Tecnológica - Espol, 33(3), 40-55. http://www.rte.espol.edu.ec/index.php/tecnologica/article/view/884 


\begin{abstract}
The Muestra Gastronómica de la Tradición Carmelita (Gastronomic Traditional Sample of Ciudad del Carmen, in English) arises from the business idea of a student of Communication and Cultural Management, as part of his course in Design and Evaluation of Entrepreneurial Projects. The origin of this project was aimed at disseminating the gastronomy of Ciudad del Carmen in Campeche, a place that receives people from all over the country and the world for its oil extraction-related activities, causing changes in the population identities which are strengthened or regenerated. The convergence aspect of this research is the resulting gastronomy. The methodological process was the researchers' empirical observation of the food consumed by people in public places such as the city's boardwalks, shopping malls, historical centers, schools, markets, etc. with the purpose of summoning the local microentrepreneurs to participate in the First Carmelite Gastronomic Show. The results of the project reported economic benefits that demonstrate its sustainability, as well as the measurement of Gastronomic Show users' satisfaction. This type of creative enterprise allows understanding how the identity around gastronomy permits culturally diverse populations to gather and enjoy a bit of their culture through the palate.
\end{abstract}

Keywords: Ciudad del Carmen, Communication and Cultural Management, Identity, Gastronomy, Cultural Industries.

\title{
Introducción
}

Habilitando a nuestros lectores para ubicar desde dónde se emite esta exploración sobre la Gastronomía Carmelita, se refiere a que el sitio de estudio se encuentra en Ciudad del Carmen, Campeche una ciudad- isla habitada por miles y miles de migrantes que vienen en búsqueda de mejores condiciones laborales, a través de la industria petrolera, que permitan una mejor calidad de vida.

Álvarez (2003) nos da un contexto de Ciudad del Carmen, brindando datos muy precisos a) uno de los 13 municipios del estado de Campeche, b) una isla, ubicada en el municipio del cual toma su nombre, mide $39 \mathrm{~km}$ de largo por $5.5 \mathrm{~km}$ en su parte más ancha y está unida por dos puentes a tierra firme que facilitan el enlace carretero entre los estados de Campeche y Tabasco, c) la ciudad y cabecera municipal del municipio del Carmen y que está asentada en la isla.

De acuerdo con el Instituto Nacional de Estadística y Geografía (INEGI) al año 2020, el Estado de Campeche tenía una población de 928 mil, 363 habitantes, y el municipio de Carmen de 248 mil 845 ciudadanos.

La Enciclopedia de la Laguna de Términos marca el año de 1979 como el inicio de la "Era del petróleo" al comenzar la explotación del campo de aceite denominado Cantarell, localizado en la sonda de Campeche. Este acontecimiento detona los movimientos migratorios, temporales o permanentes, de extranjeros y mexicanos de diferentes partes de la República a Ciudad del Carmen. Debido a la movilización de empresas en el complejo proceso de extracción del petróleo, canadienses, norteamericanos, venezolanos, brasileños, chinos, haitianos entre otras nacionalidades, son parte integrante del complejo social carmelita, lo mismo que mexicanos de diferentes estados, destacando Tamaulipas, Veracruz y Tabasco.

Desde este contexto nos permitimos plantear el proyecto motivo de esta investigación. El mismo se gesta como parte del curso Diseño y Evaluación de Proyectos Emprendedores del programa Educativo de Comunicación y Gestión Cultural que se imparte en la Universidad Autónoma del Carmen, se creó la empresa Ikal Ha (Espíritu del Agua) con el objetivo de 
brindar a la comunidad carmelita soluciones culturales para fortalecer la identidad sociocultural de la isla. Esta empresa nació a través del modelo de un plan de negocios que permitiera hacer sostenible a la misma.

El primer servicio cultural organizado por dicha empresa fue la Muestra Gastronómica de la Tradición Carmelita. La justificación de esta firma creativa se hizo evidente debido al contexto sociocultural de Ciudad del Carmen, la cual es una isla cuya principal actividad económica es la petrolera, debido a esto, a la ciudad llegan de paso o a vivir por diversos lapsos de tiempo, personas no solo de las diferentes entidades federativas de México, sino del mundo, lo que genera un proceso identitario de una dinámica particular.

Es común encontrar en la ciudad alimentos veracruzanos, tabasqueños, sinaloenses, poblanos, oaxaqueños, así como argentinos, venezolanos, cubanos, entre muchos otros, por supuesto, teniendo su lugar de honor, la gastronomía carmelita con la elaboración de platillos obtenidos del mar.

En este contexto nos permitimos proyectar la creación de la empresa que dio origen a la Muestra Gastronómica de la Tradición Carmelita, la primer tarea fue comprender el proceso identitario del oriundo de la isla, de esas personas que son nativas de Ciudad del Carmen, pero que también se caracterizan por su alto grado de hospitalidad, ya que la isla durante muchos años ha sido visitada por diversas personas del país y del mundo debido a sus distintas etapas económicas, tales como: la extracción y elaboración del chicle, maderas preciosas, la copra, la industria camaronera y actualmente la petrolera.

Por otra parte, se recuerda que la gastronomía mexicana fue declarada Patrimonio Cultural Inmaterial de la Humanidad.

En el año 2010, la gastronomía mexicana fue declarada Patrimonio Cultural Inmaterial de la Humanidad por la Organización de las Naciones Unidas para la Educación, la Ciencia y la Cultura (UNESCO). Gracias a esto, se enalteció que la cocina en México sea considerada elemento crucial de identidad nacional, debido a su historia, creatividad, diversidad y trascendencia. Fernández E. (2 de diciembre de 2016).

Este hecho ha permitido cobrar un nuevo sentido de identidad al ser mexicanos, de esta forma cada estado de la República enaltece su herencia culinaria, entrelazándose con identidades asentadas en las comunidades, de esta forma en Ciudad del Carmen las personas igual degustan un tradicional pan de cazón de la zona, unos hot dogs, unos panuchos, una pizza, unas suaves, unos camarones al coco, al igual que una hamburguesa, así surge la riqueza cultural manifiesta en la gastronomía con la apropiación identitaria a través de alimentos y su reelaboración, que han llegado con la migración y la consecuente diversidad cultural.

Como parte del andamiaje teórico y el estado de la situación del tema de identidad con respecto a la gastronomía visto como un elemento cultural que construye al individuo en una comunidad, se toman diversas posturas teóricas que nos permiten comprender el tan complejo concepto.

Entendemos por identidad o en plural como identidades a estos procesos inacabados y continuos mediante los cuales los individuos se elaboran en su diario acontecer en respuesta a la imperiosa necesidad de los seres humanos de ubicar su lugar en el mundo, su relación con los otros agentes y con ello dar razón a su existencia. 
En Discourse Analysis, Paltridge (2012) profundiza en esta idea acotando que la manera en que vestimos, los gestos que empleamos y las formas en las que actuamos e interactuamos con los demás influyen en cómo exhibimos nuestra identidad social. Para fines de este trabajo, nosotros hacemos más "elástica" esta concepción y la extendemos a las prácticas culinarias y alimenticias de los individuos, que moldean y conforman la identidad social. Inmersos en un universo multicultural como el nuestro es imposible escaparse de la gastronomía de los migrantes de diferentes estados de la República Mexicana y de diferentes partes de mundo.

En diferentes zonas de esta pequeña isla podemos degustar lo mismo un zacahuilt de la región norte del estado de Veracruz, que unas tlayudas del estado de Oaxaca, unos tamales de chipilín del vecino-hermano estado de Tabasco que unos tequeños o unas arepas venezolanas. Si bien cada uno de nosotros estamos conscientes de que nuestras identidades son socialmente ubicadas porque representamos y reconocemos las características del país y del estado de la república del cual somos originarios, es inevitable dejarnos seducir por la amplia oferta gastronómica de los diferentes escenarios en los cuales nos desenvolvemos, la continua seducción a los sentidos corpóreos a través de los olores y sabores disponibles en la isla es inevitable. Y no es que dejemos de un lado la identidad de nuestros orígenes, pero caemos en el juego de la seducción y somos reinventados en el diario acontecer jugando el cautivador juego de los sabores.

Por otra parte, Infante (2008) subraya esta continua construcción del cuerpo, estableciendo al mismo tiempo su concepción de discurso:

El discurso no es algo que se inscribe sobre el cuerpo, ni algo que se produce en referencia a él, sino más bien, el proceso mismo mediante el cual el cuerpo se materializa... el discurso es incorporado, encarnado o convertido en cuerpo; cuando el discurso es reiterado performativamente por un cuerpo, siendo esta "performación" la que le da al cuerpo una existencia, agencia e identidad que luego parecieran existir previo al discurso. (p.17)

Esta idea de discurso como cuerpo y cuerpo como discurso enfatiza el proceso continuo en el que el cuerpo se ve inmerso por el hecho de existir, configurarse y significar y resignificarse para sí mismo y para el otro, reclamando un derecho propio al disfrute y concediendo agencia a los elementos gastronómicos varios y a los vínculos que establecemos con los proveedores de las materias primas, productores-vendedores y consumidores.

Con las dos propuestas teóricas vistas hasta ahora, vemos diferentes formas y escenarios en la construcción de la identidad en torno al tema de la gastronomía, de esta manera Preciado (párrafo dos) expresa la idea de pensar el cuerpo como una "somateca" o archivo de múltiples procesos de construcción que lleva implícitos los resultados de la acción de diversos dispositivos de poder que resultan en la configuración de las diferentes "ficciones políticas" que nos conforman y nos constituyen. Entre estos múltiples lenguajes y técnicas de poder se establecen diferentes tipos de relaciones y conflictos que toman la forma de vida, que se "somatizan". Es inevitable ser ajeno a la realidad social del paisano Oaxaqueño que se acerca a la isla con la empresa de ofrecer su producto con el fin de ofrecer una mejor vida a sus familiares, ni qué decir del hermano venezolano que se finca en la isla, muchas veces a pesar de haber perdido su contratación en una empresa petrolera por falta de recursos, pero que tiene un mejor estilo en esta tierra que lo cobija y a la que ofrece sus manjares. No, los consumidores no son ajenos, se deleitan en los sabores, se hermanan con las historias de vida del migrante que ofrecen su gastronomía. 
La postura anterior conduce a Hallensleben (2011), y a pensar enfáticamente en la naturaleza performativa del cuerpo. El autor convoca a reflexionar sobre el ser y tener un cuerpo, él manifiesta que se está continuamente en proceso de creación, de producción, uno es y se hace a cada momento reclamando el espacio que el mismo cuerpo ocupa y de esta forma, agregamos, la identidad no es ajena a este asunto, por ello podemos adoptar de forma tan natural nuevos procesos identitarios al integrar en las tradiciones nuevas maneras de degustar la gastronomía de nuestro, país, localidad y del mundo.

Las técnicas etnográficas de observación participante que orientan la perspectiva del trabajo de investigación se remiten a Soja (1989) quien propone pensar la geografía en términos diferentes y recomponer la noción de "espacio". Además de un "primer espacio" en el que se ubica lo que se percibe y de un "segundo espacio" asociado a lo que se concibe, se advierte la posibilidad de un "tercer espacio" que, si bien es "otro", no excluye, sino que contiene a los otros dos. En este tercer espacio, Soja privilegia la construcción del ser centrado en lo vivido:

Cada una de estas dimensiones existenciales abstractas cobra vida como una construcción social que da forma a la realidad empírica y es simultáneamente moldeada por ella. Así, el orden espacial de la existencia humana surge de la producción (social) del espacio, la construcción de geografías humanas que reflejan y configuran el ser en el mundo. (p.25)

A partir de esta aportación se invita a reivindicar la conciencia del "estar vivo", acentuar lo vivido como una dinámica de colección de procesos incorporados, que se viven, se experimentan y manifiestan a través de los cuerpos. En este sentido, esta concepción de un tercer espacio confluye con nuestra apuesta por visibilizar los procesos performativos de los cuerpos inevitablemente inmersos en una dinámica social a partir de la cual se elaboran a sí mismos construyendo la propia realidad, en un flujo continuo e inacabado en el que tienen especial cabida la imaginación, las recomposiciones y los cuestionamientos al orden imperante, a las prácticas sociales, otras diferentes a las del propio marco de referencia.

Al reflexionar sobre esta particular dinámica impuesta por la naturaleza de las actividades en la isla y dotada de nuevas herramientas teóricas a partir de este curso, es que reflexionamos, siguiendo a Borden (2002) "Mirar" la arquitectura, entonces, es... explotar toda la noción de tiempo y espacio; requiere comprender con múltiples ideas e intelectos, con todo el cuerpo, con el corazón y la mano" (p. 9).

Las reflexiones a partir de esta lectura invitan al compromiso al involucramiento, tanto físico como intelectual y emocionalmente con el entorno en el que tenemos nuestra casa en la isla del Carmen, Borden (2002) expresa "Contemplar el espacio con todo el cuerpo y todos los sentidos, no solo con los ojos y el intelecto, permite una mayor conciencia de los conflictos y por lo tanto de un espacio que es Otro" (p.11).

Los autores sugieren que no es común que reflexionemos sobre estos espacios que son diferentes de nuestra propia corporalidad y que nos invitan a orientarnos a partir de referentes sensoriales que no siempre tenemos activados o en estado de alerta. ¿A qué huele mi entorno? ¿Cuáles son los diferentes matices que nos ofrece la brisa marina dependiendo de las condiciones climáticas? ¿Cuáles son los sonidos que se escuchan en mi barrio? ¿Podría distinguir los sonidos propios de la naturaleza de aquellos que resultan de las actividades humanas cotidianas? ¿Se escucha diferente un domingo de un lunes? 
Borden, et al (2002) Las ciudades son sistemas complejos de representaciones, en los que el espacio y el tiempo se entienden y experimentan en forma de representación" (p.14). De esta forma el autor convoca a tomar en cuenta las experiencias del yo narrador habitante en determinado lugar, a través del proceso de "verbalizar" nuestra experiencia con el lugar, las construcciones, los elementos de la naturaleza, los olores y los platillos dejan de ser objetos y se vuelven los lugares de negociación epistemológica y social.

Presentamos las principales posturas teóricas que nos permiten tener una perspectiva más amplia de cómo la identidad tiene que ver con el sentido de tomar conciencia del propio proceso identitario -que es de por sí complejo de estudiar y comprender- pero, que al momento de configurarse en colectivo, en comunidad, puede derivar en las coincidencias culturales que nos hacen sentir orgullo de determinados hechos simbólicos, por ejemplo: la resultante gastronomía de la ciudad, que hace evidentes esos procesos identitarios.

Este proyecto nació también con el objetivo de demostrar que los emprendimientos culturales son factibles y sostenibles como cualquier otro giro con visión empresarial, se tiene la idea equivocada que los proyectos culturales son empresas que no generan aportación económica a sus creadores, esta, es una idea equivocada.

Al respecto la Cámara Nacional de la Industria de Restaurantes y Alimentos Condimentados (CANIRAC) en su informe "Todo sobre la mesa" (2019) dio a conocer que las unidades económicas dedicadas a la preparación de alimentos y bebidas en el 2018 generaron 247 mil 360 millones de pesos, lo que representó el 1.1 \% del PIB total del país.

Por otra parte, el Instituto Nacional de Estadística y Geografía en su informe (2019) nos da a conocer que su definición de cultura para el aspecto económico la representa de la siguiente manera.

Considera las recomendaciones y estándares estadísticos internacionales como el Sistema de Cuentas Nacionales 2008 de las Naciones Unidas, OCDE, entre otros, así como el Marco para Estadísticas de Cultura de la UNESCO, y la Guía Metodológica para la implementación de las Cuentas Satélite de Cultura en Iberoamérica del Convenio Andrés Bello. INEGI (2019)

Es así como de acuerdo con este organismo la cultura aportó, en 2019, el 3.1 \% al PIB del país, lo que representó en pesos mexicanos 724 millones 453 mil pesos. Lo que demuestra el impacto económico que representan estos rubros como empresas creativas. Lo emprendimientos culturales cumplen con el rigor de la visión empresarial, se constituyen por un proceso profesional que permite su creación, implementación y evaluación.

\section{Metodología}

Para realizar la Muestra Gastronómica, se realizó un proceso de observación empírica por parte de cada uno de los integrantes del equipo, quienes se dieron a la tarea de analizar, según sus propias experiencias, aquellos platillos y/o alimentos que, siendo originarios o no, representan la cultura o tradición de los habitantes de Ciudad del Carmen.

Se observaron distintos grupos: familias, jóvenes, adultos, y como en distintos momentos del año o del día, acostumbran a consumir ciertos alimentos, y como muchos de estos, sin ser originarios del lugar, ya son un referente cultural del mismo. 
Esta observación permitió organizar una dinámica de focus group en el que participaron siete jóvenes de entre 19 y 25 años, estudiantes todos del tercer semestre de la Licenciatura en Comunicación y Gestión Cultural de la UNACAR, quienes integrarían el Comité Organizador de la Muestra Gastronómica. En el grupo, a manera de lluvia de ideas se fueron discutiendo algunos aspectos a considerar para elegir los platillos que habrían de tomarse en cuenta para la Muestra, además de los platillos locales, aquellos que son parte de la vida cotidiana o de las costumbres de los carmelitas, por ejemplo, es costumbre que después de una noche de "antro" cenar a altas horas de la madrugada hot dogs o "perros calientes" en alguno de los puestos más antiguos de la ciudad, también es parte de las costumbres comprar elotes o esquites en las tardes para caminar por el malecón. También se tomaron en cuenta lugares o restaurantes a los que se lleva a comer o almorzar a algún familiar o conocido foráneo cuando está de visita en la Isla.

Después de este diálogo se elaboró una lista general de restaurantes y pequeños negocios que serían invitados a participar en la Muestra, generando una lista de más de 30 participantes.

Posteriormente se redactó la convocatoria, la cual fue emitida un mes y medio antes de la realización del evento, tomando en cuenta los requerimientos que cada participante debería cumplir, en la convocatoria se detallaban aspectos técnicos de la Muestra: requisitos de participación, la mecánica de inscripción y la mecánica de elección del platillo ganador. Se tomó como criterio segmentar la muestra en tres categorías: Pescados y Mariscos, Alimentos en General y Dulces y postres.

Se procedió a distribuir la convocatoria a los empresarios que se pudieran interesar en participar, entregando personalmente la misma, junto a una invitación a participar, en la mayoría de los casos esta visita invitó a un diálogo más profundo con los representantes de los restaurantes para explicarles el contexto de la actividad, explicándoles que la muestra era un espacio en el que podían dar a conocer sus platillos y difundir sus establecimientos.

Adicionalmente se dio promoción a la convocatoria a través de las redes sociales creadas ex profeso para la Muestra y la de la Universidad.

Finalmente se decidió establecer la cuota de recuperación para los visitantes que para los niños fue de $\$ 15$ pesos mexicanos y para los adultos $\$ 25$ pesos mexicanos, los que les dio derecho a degustar todas las pruebas que pusieron para tal fin los expositores, así como para el disfrute de las actividades programadas (presentaciones artísticas y conferencia).

\section{Análisis de resultados}

Después de este proceso de convocatoria y selección, se logró contar con la participación de 19 expositores, ocho en la categoría de pescados y mariscos, cuatro en alimentos en general y siete en dulces y postres, lo que nos llevaría a realizar el trabajo etnográfico de vivir el entorno y de identificar los elementos más significativos de la gastronomía local:

En la categoría pescados y mariscos la mayoría de los participantes presentaron platillos elaborados principalmente con camarones, en sus diversas presentaciones, también destacó el pulpo, ostión y otros mariscos de la región, incorporando otros ingredientes locales como el coco, pero también embutidos y otros ingredientes no locales.

En la categoría de alimentos en general participaron expositores de antojitos de la región, como empanadas, panuchos, suaves -que son alimentos muy característicos de la 
península- también se integraron tamales de masa colada, que son muy comunes de encontrarse en puestos ambulantes en puntos diversos de la ciudad y que ya se volvieron referencia para los habitantes de esta isla. Se contó la participación de una pollería que compartió los complementos con los que acompaña los pollos asados, como espagueti, ensalada de coditos, ensalada de pollo, puré de papa, que también son considerados alimentos típicos por considerarse tradicionales en los rezos o actividades de relacionadas a las prácticas religiosas populares en la localidad.

En la categoría de postres, destacaron los dulces locales como merengues y las tradicionales cremitas rosas, también alimentos que sin ser de la ciudad ya gozan de sólida reputación respaldada por una larga tradición como son los churros y los esquites, que han sido transformados a lo largo de los años, pero que son un referente de antojitos en las tardes de paseo familiar. También se permitió la participación de nuevas propuestas como panes y pays miniaturas que son postres que gozan de popularidad entre la comunidad universitaria UNACAR, donde se comienzan a destacar fuertemente. También se contó con la participación de "Helados Parcero", negocio local de helados de frutas naturales y paletas, que se ha convertido en un fuerte referente en todo el estado de Campeche.

La muestra gastronómica de la tradición Carmelita fue realizada el 22 de noviembre de 2017 en el Guanal Museo Universitario de Ciencias y Artes de la UNACAR, a las 12 horas.

A la par de la muestra gastronómica, se presentaron otras manifestaciones artísticas relacionadas con la cultura de la isla: danzas Mayas, bailes tropicales, artes plásticas, música en vivo.

La danza Maya fue elegida como número de apertura de la muestra como elemento que representó el antecedente de la cultura de la región peninsular, el Ballet Folclórico Ixchel, fue el encargado de presentar este número. Se presentaron posteriormente bailes tropicales identificados como música típica de la ciudad, ritmo muy popular y animado que suele ser común en convivencias familiares o fiestas entre amigos, de esta manera se ofreció a los asistentes elementos de entretenimiento y acercamiento a la música local, lo que permitió crear una atmosfera cultural con un fuerte distintivo de la Isla.

Antes de realizar la clausura de la Muestra, un dúo de cuerdas de la Escuela de Música de la UNACAR deleitó a los asistentes con música regional, pero en ritmos más calmados y clásicos, ofreciendo a los asistentes una nueva propuesta de música.

Para evocar al intelecto de los participantes, se invitó a la Dra. María Rosano, quien impartió una conferencia titulada "Historia de la Gastronomía Carmelita", en la que destacó los elementos que históricamente han ido influenciando en la gastronomía de la ciudad.

Las artes visuales fueron incluidas desde dos aspectos, primero por el lugar del evento que cuenta con una exposición permanente de la historia de la Ciudad, con obras plásticas de distintos autores, y también en los premios entregados a los participantes, que consistió en obras plásticas del Mtro. Leonel Cortés Zepeda, siendo cocos pintados al óleo con platillos típicos de la región: Pan de Cazón, Pescado Frito y Camarones al Coco.

Los asistentes a la muestra tuvieron la oportunidad de elegir, de entre todos los participantes, el que más les gustó, votando mediante una papeleta que se repartió y que depositaban en una urna, en esta misma papeleta también se incluyó una pequeña encuesta de satisfacción del evento. Dicha papeleta contemplaba dos secciones: la primera preguntando la 
opinión del asistente acerca de la organización del evento, la segunda preguntando acerca de su opción favorita en cuanto a cada categoría de los alimentos de la Muestra.

Los votos fueron contabilizados por el equipo de staff, y antes de clausurar la Muestra se dio a conocer a los ganadores los cuales recibieron un presente.

En lo que respecta al hecho de realizar una actividad empresarial cultural sostenible, después de la inversión realizada por el grupo organizador, se obtuvo un rendimiento de $\$$ 7,500 pesos mexicanos, los cuales se consideran con un logro importante después de todo el trabajo realizado de búsqueda de patrocinios y apoyos, el evento resultó económicamente redituable.

Como se expuso en los apartados anteriores, en donde se sustenta el andamiaje teórico y metodológico, se muestran ahora los resultados cuantitativos de la satisfacción de los usuarios que asistieron a la muestra gastronómica.

En la Figura 1 se observa que 117 asistentes que respondieron el instrumento aplicado externaron un grado de satisfacción de excelente lo que cubría los rubros de organización, calidad de los productos de los expositores, entre otros aspectos:

\section{Figura 1}

Grado de satisfacción de los asistentes a la muestra gastronómica

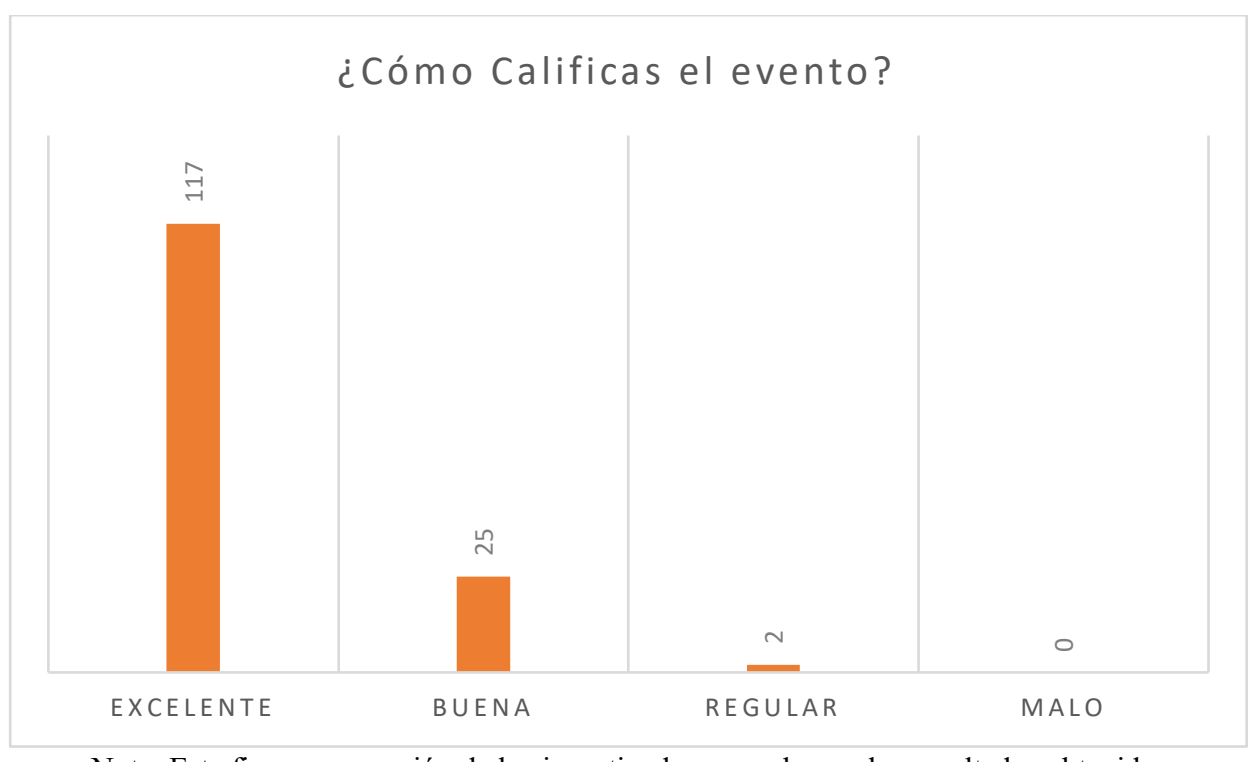

Nota: Esta figura es creación de los investigadores con base a los resultados obtenidos

Por otra parte, en la Figura 2 se observa que el área que más disfrutaron los asistentes fue la visita a los expositores, seguido de la degustación de los platillos.

En la Figura 3 se observa la opinión de los asistentes respecto a las actividades de la siguiente muestra, teniendo el mayor número de propuestas la presentación de grupos artísticos y talleres gastronómicos.

En la Figura 4 se les preguntó sobre el costo de la cuota para ingresar a la muestra, en donde el $67 \%$ indicó que le pareció excelente el costo y un $24 \%$ dijo que la cuota fue buena. 


\section{Figura 2}

Actividad de mayor agrado de parte de los asistentes

¿Qué es lo que más te gustó de la muestra?
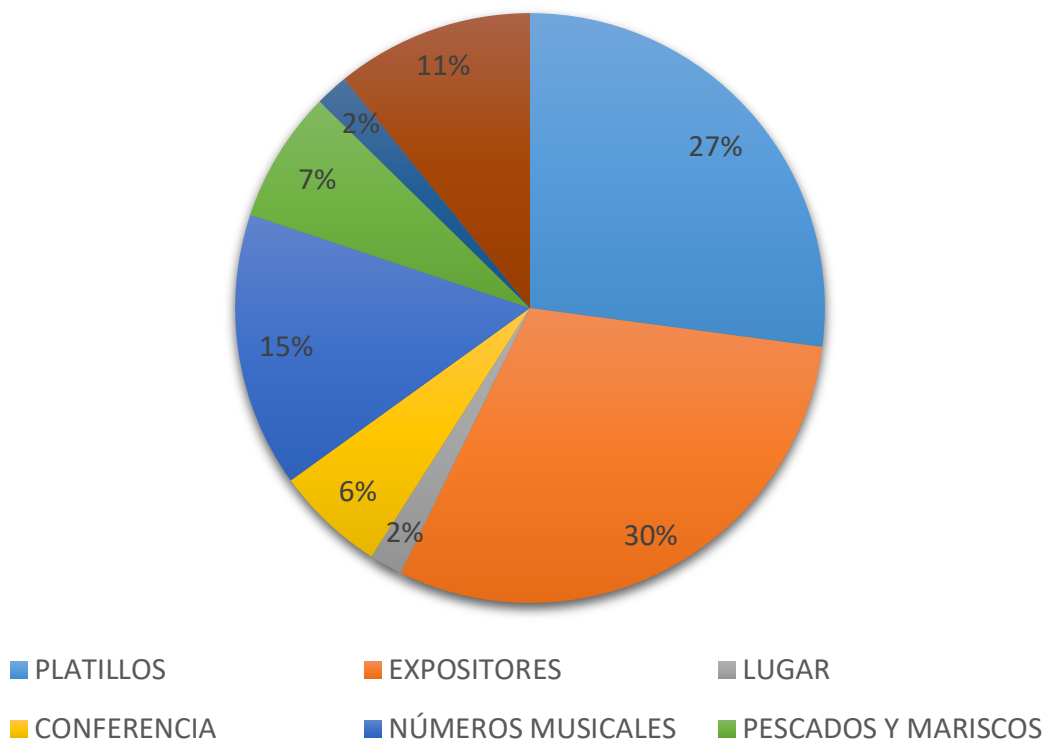

aLIMENTOS EN GENERAL — DULCES Y POSTRES

Nota: figura realizada por los investigadores a partir de los resultados

\section{Figura 3}

Actividades solicitadas para la siguiente edición:

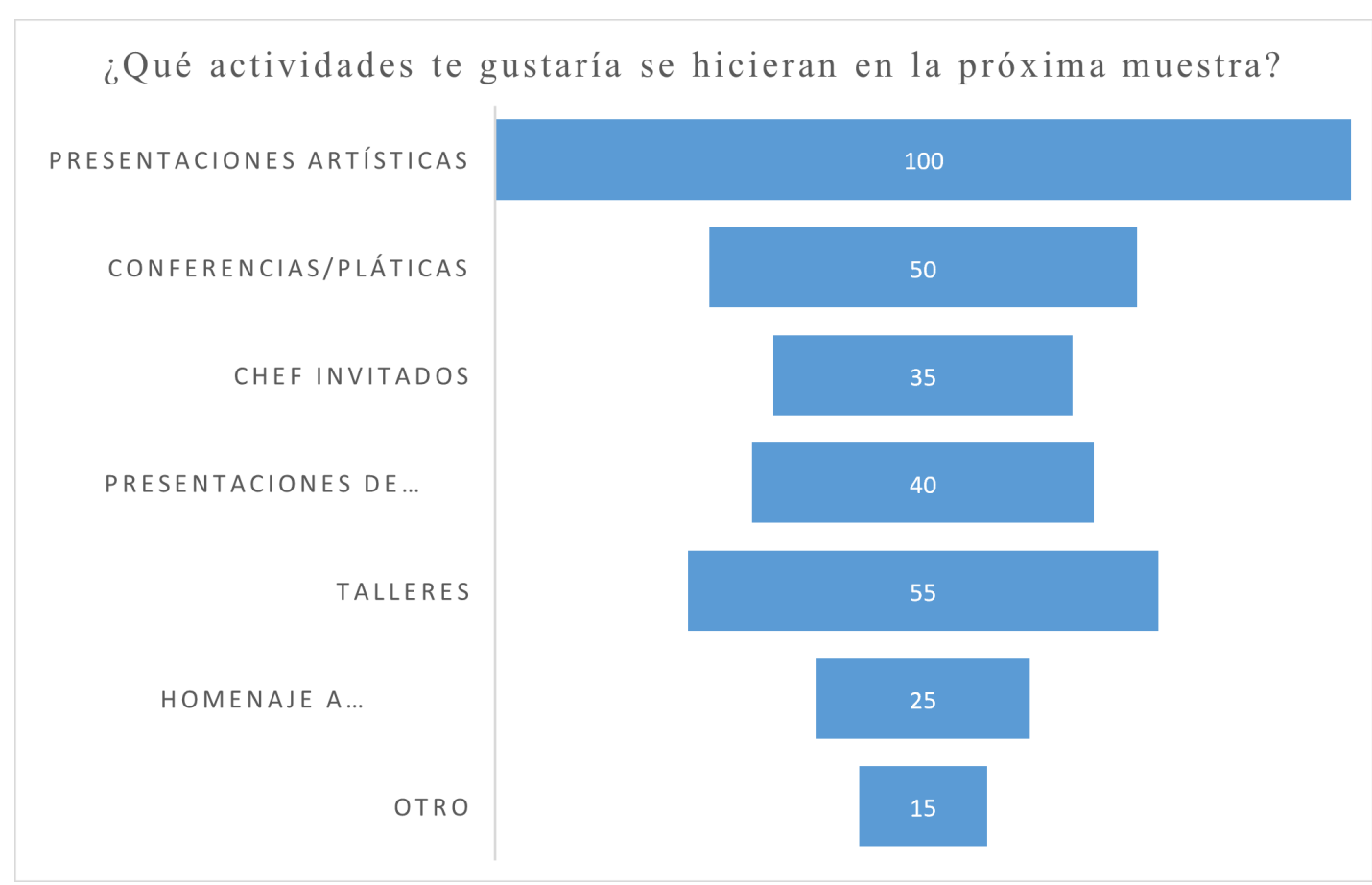

Nota: figura realizada por los investigadores a partir de los resultados 


\section{Figura 4}

Opinión de los asistentes con respecto a la cuta de recuperación

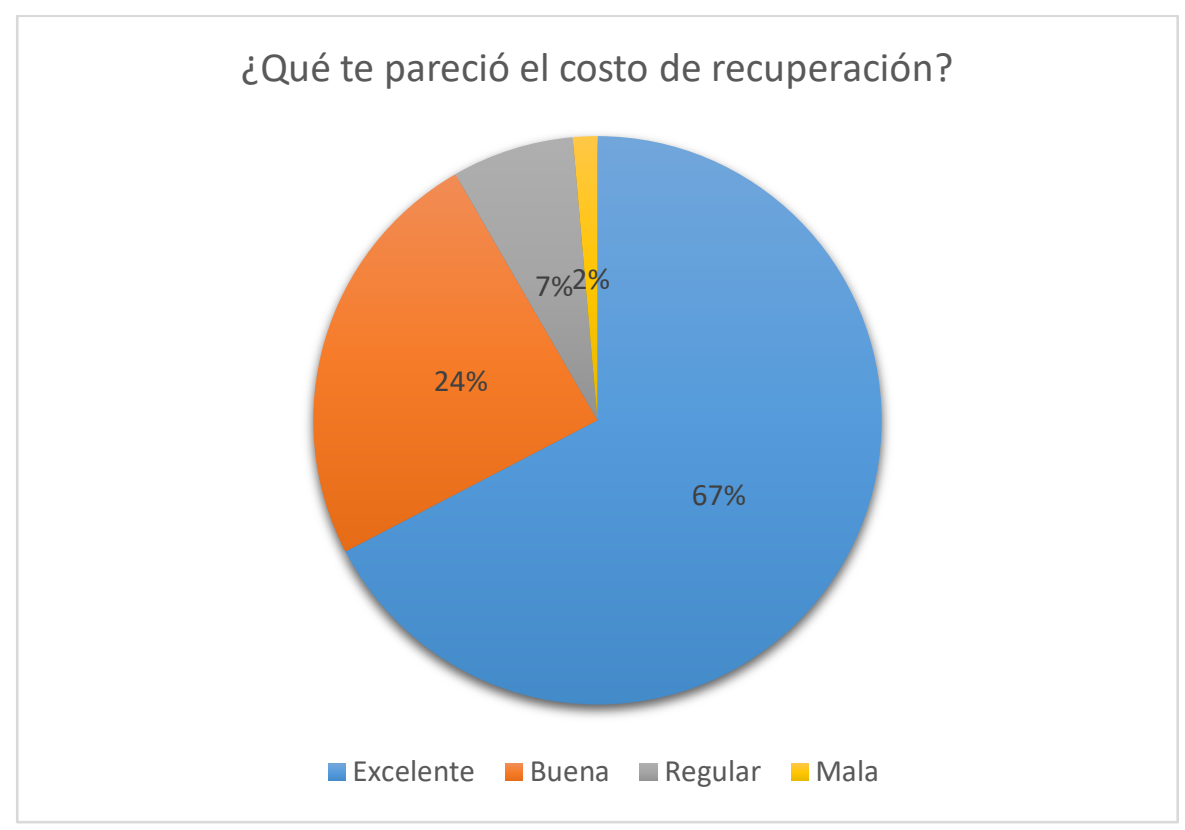

Nota: figura realizada por los investigadores a partir de los resultados

Uno de los aspectos más importantes fue identificar los giros que tuvieron el mayor número de usuarios satisfechos con la presentación de los platillos que se degustaron, de esta forma en el giro de restaurantes de mariscos, el porcentaje más alto fue del $19 \%$ de acuerdo con los que respondieron, fueron 23 comensales que eligieron como el de mejor calidad a "Restaurante Cayo Arcas", pero, si observamos el gusto del resto de los asistentes es bastante uniforme de forma positiva. Véase Figura 5.

\section{Figura 5}

Los mejores restaurantes de pescados y mariscos de Ciudad del Carmen

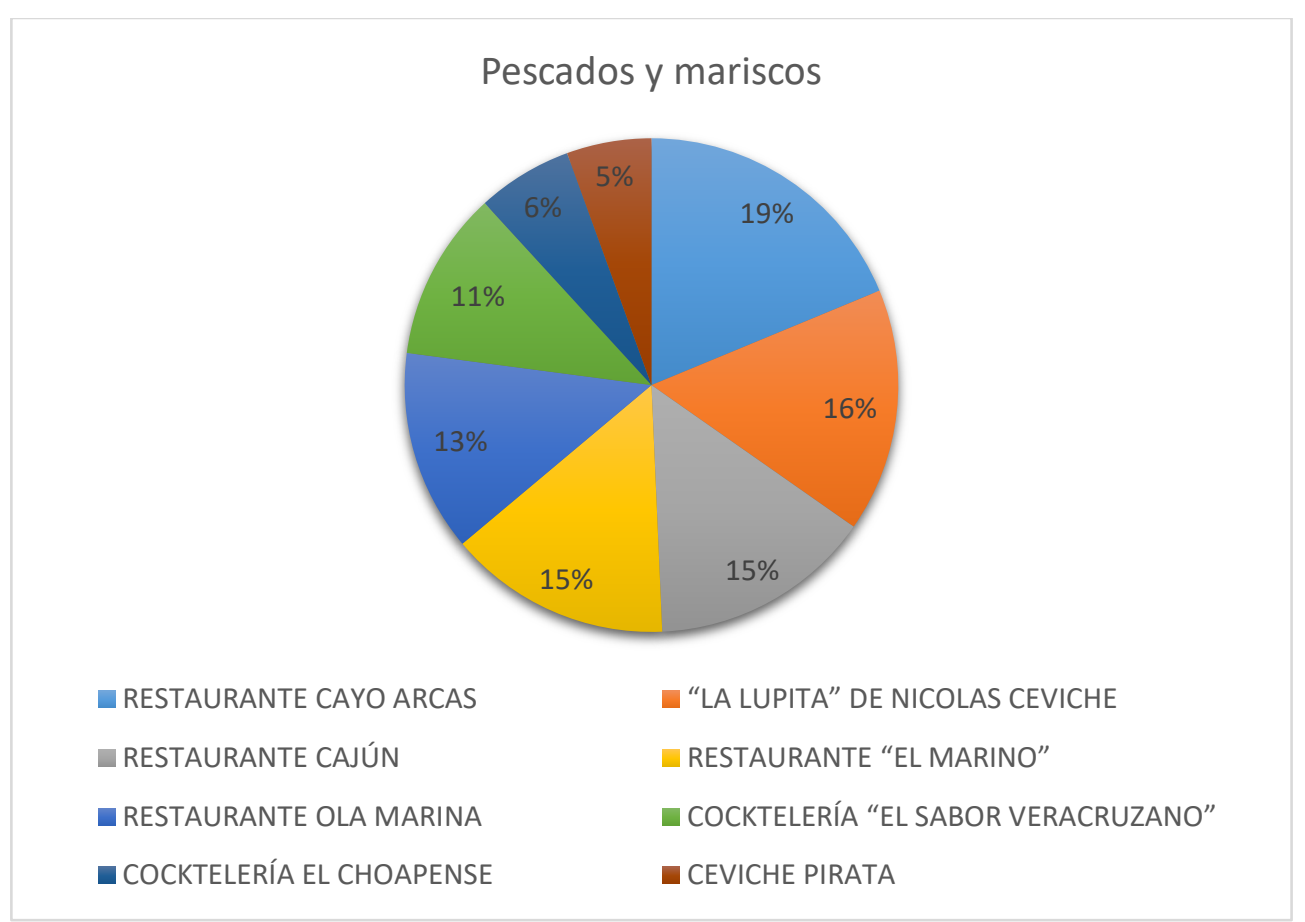

Nota: figura realizada por los investigadores a partir de los resultados 
En el rubro de Alimentos en general, los asistentes externaron su gusto por los tamales "El Milagro de Chuina" y por los antojitos regionales que ofreció "Doña Licha", obsérvese la Figura 6.

\section{Figura 6}

Los alimentos dentro del rubro general de mayor gusto por parte de los asistentes

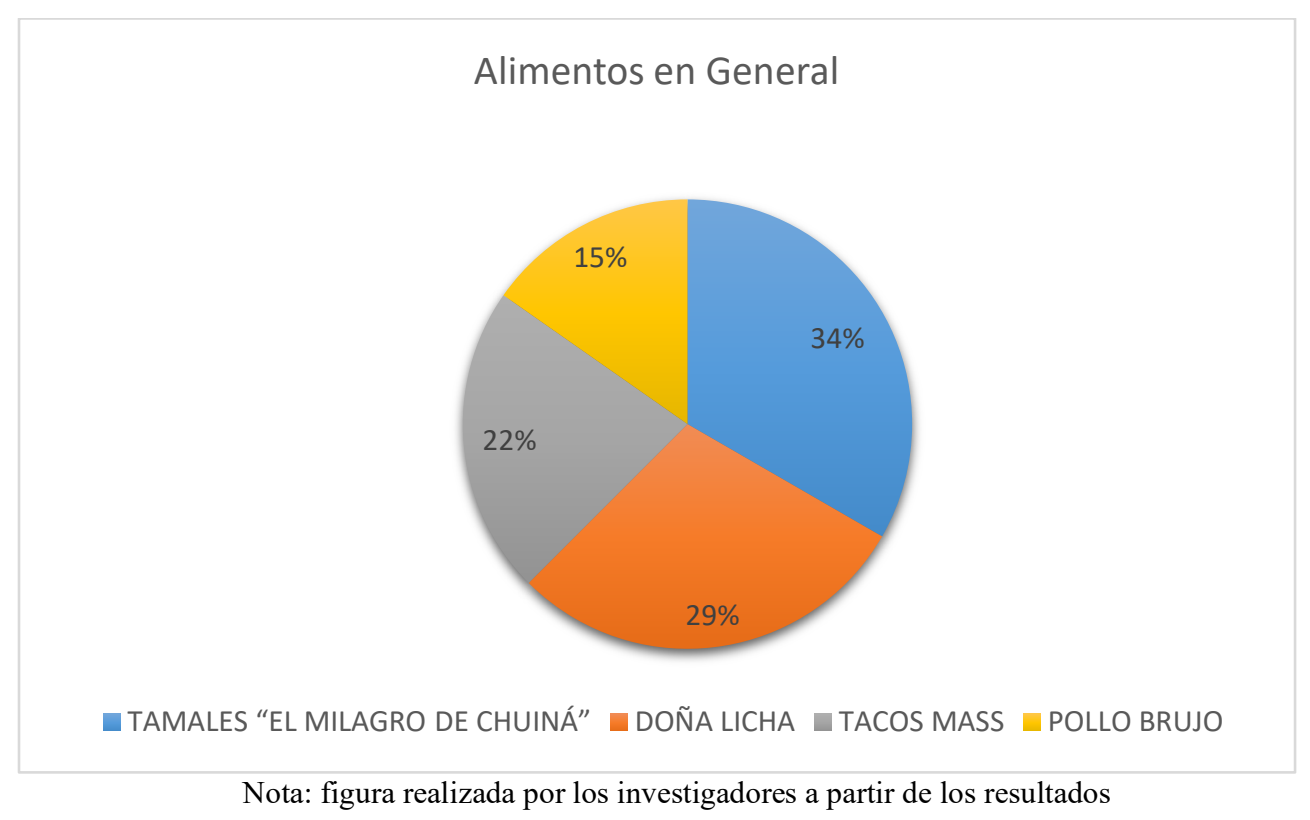

En el tercer rubro de la Muestra en lo que se refiere a postres y dulces, los asistentes dieron el mayor porcentaje al giro de negocio llamado "Paysito", seguido de" Merengues Adda", de acuerdo con la Figura 7 el gusto estuvo bastante equitativo entre la diversa variedad de la Muestra.

\section{Figura 7}

Preferencia de los asistentes en cuanto a los expositores de dulces y postres

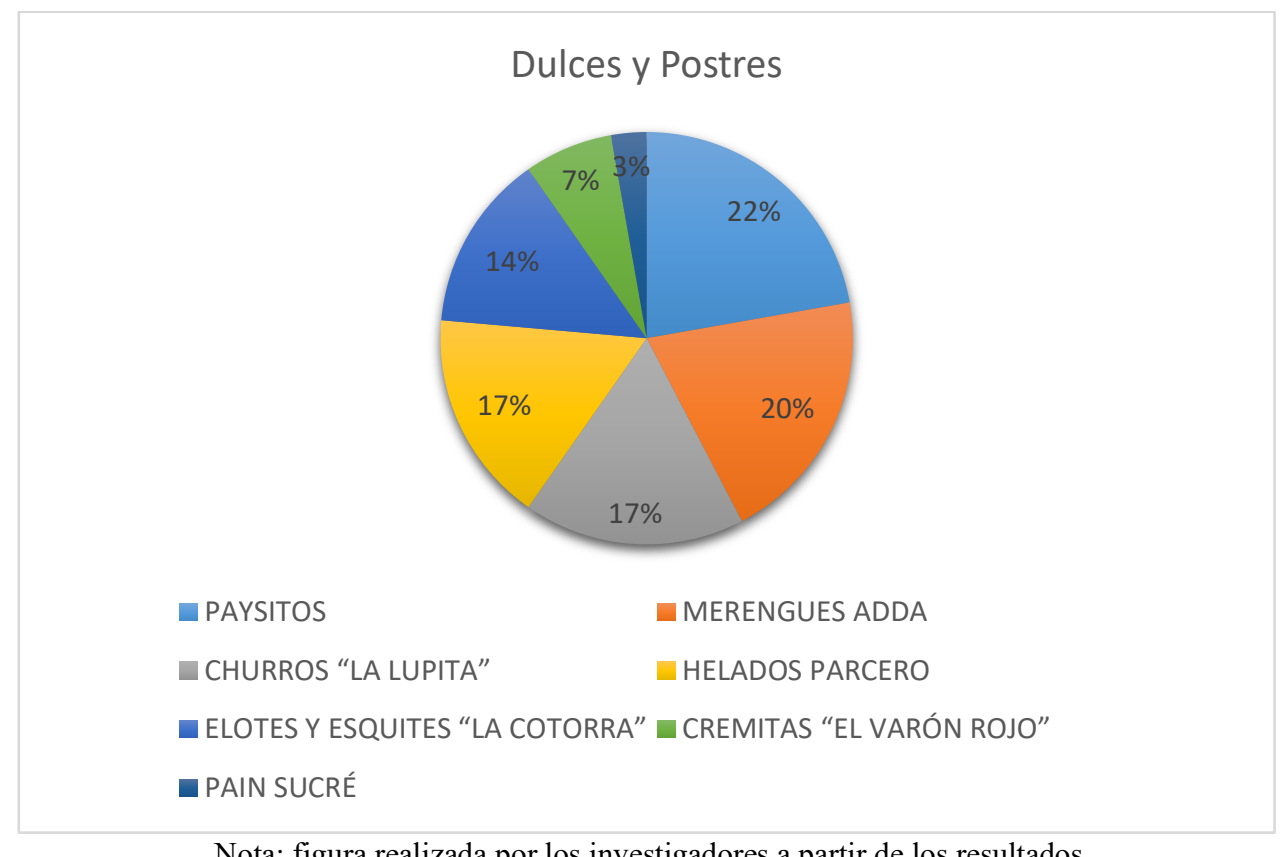

Nota: figura realizada por los investigadores a partir de los resultados 
Durante la evaluación de la Muestra de la Tradición Gastronómica Carmelita se le pidió el grado de satisfacción a los expositores los cuales respondieron un instrumento, obteniéndose los siguientes resultados.

En la Figura 8 se observan las respuestas, en donde 16 de los 19 expositores externaron una percepción excelente de la actividad.

\section{Figura 8}

Grado de satisfacción de los expositores con respecto a la Muestra de la Tradición Gastronómica Carmelita

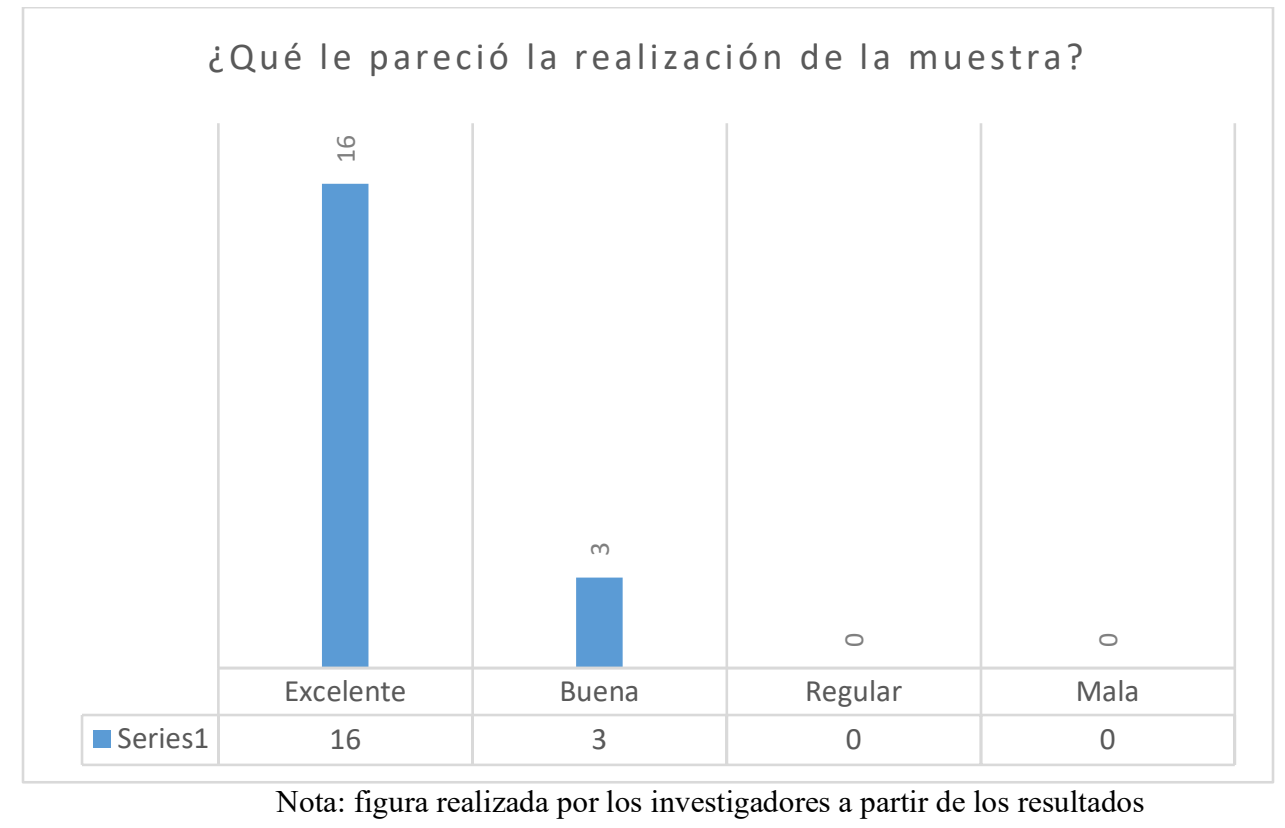

Por otra parte, al preguntárseles si volverían a participar en una siguiente edición, el $100 \%$ de los entrevistados externaron una respuesta afirmativa (Figura 9).

\section{Figura 9}

Respuesta de los expositores para participar en una siguiente edición

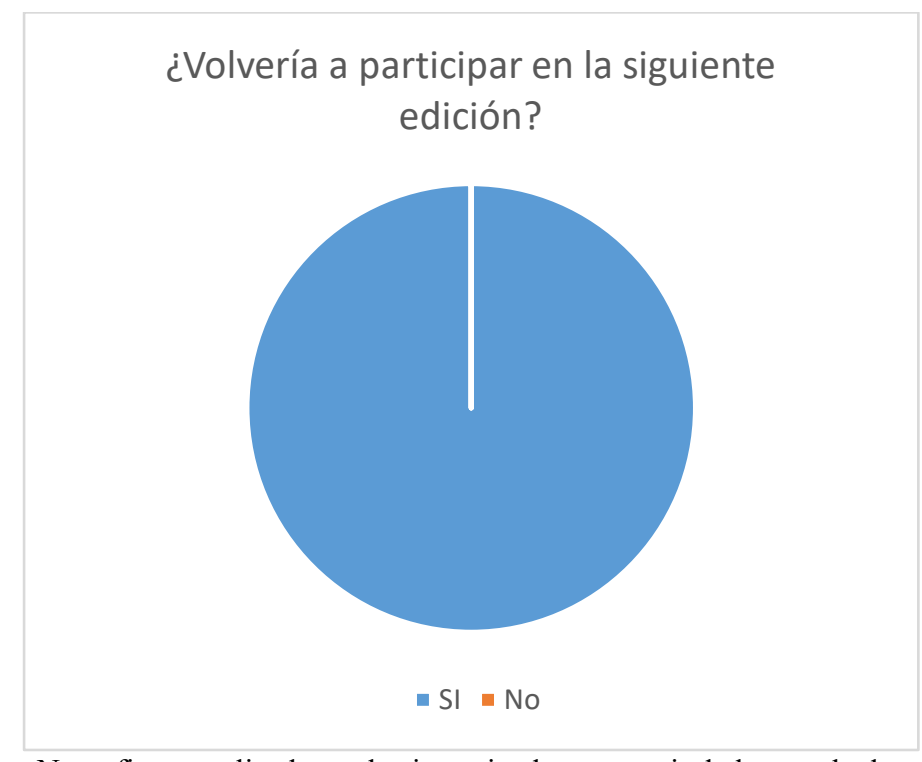

Nota: figura realizada por los investigadores a partir de los resultados 
En cuanto a la pregunta respecto a la organización en general de la muestra 13 de los expositores externaron una calificación excelente y tres buena (Figura 10).

\section{Figura 10}

Respuesta de los expositores con respecto a la organización en general

¿Cómo considera la realización del evento?

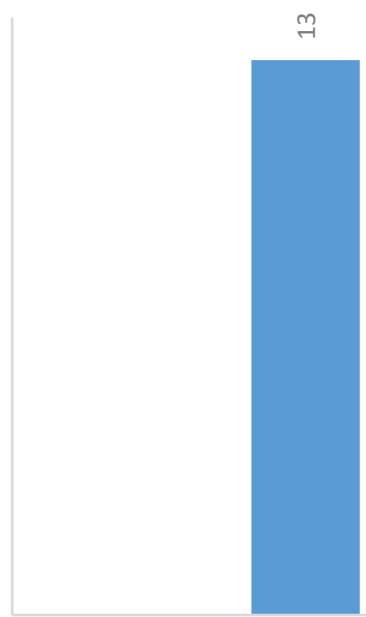

EXCELENTE

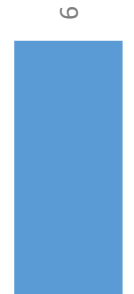

$B \cup E N A$

Nota: figura realizada por los investigadores a partir de los resultados

En la Figura 11 los expositores opinaron a las áreas de mejora de la muestra, expresando que un lugar más amplio sería lo ideal.

Figura 11

Opinión de los expositores para las mejoras de la siguiente edición

¿Qué nos sugiere mejorar para la próxima edición?

14

12

2

0

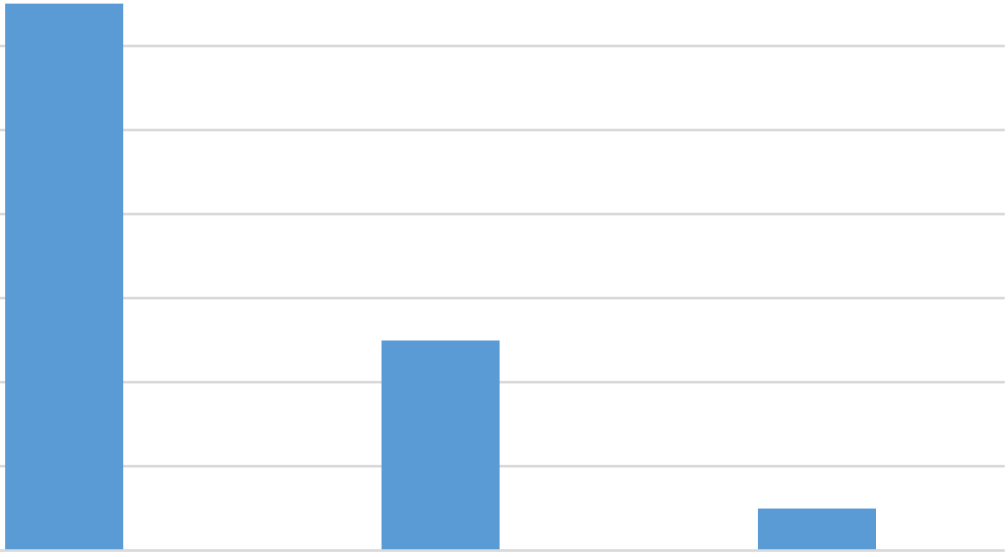

Mayor espacio

Mayor difusión

Más cupones

Nota: figura realizada por los investigadores a partir de los resultados 


\section{Discusión de resultados}

Después de medir lo que cuantitativamente se estableció y tomando como referencia a los autores indicados en la introducción, la primera intención de la muestra fue demostrar el conocimiento adquirido a través de una asignatura que brinda y apoya el desarrollo de competencias profesionales como es el Diseño y Evaluación de Proyectos Emprendedores con perfil cultural. La metodología seleccionada da fe de que el rigor científico de la mano de la visión empresarial mediante el emprendedurismo es una forma de mover la economía de las pequeñas comunidades.

Las propuestas teóricas que se plantean en el trabajo no fueron seleccionadas como forma de contraposición, al contrario, se eligieron cuidadosamente para demostrar cómo la reconfiguración de la identidad cultural a través de la comida puede ser comprendida de diversas formas.

Paltridge (2012) externa que las identidades se van moldeando de acuerdo al entorno social y para el caso de este trabajo de investigación se toma la gastronomía como referencia, en los tres rubros establecidos en la muestra que fueron de pescados y mariscos, antojitos y alimentos diversos y postres y dulces, la mayoría de los asistentes externaron una posición actitudinal positiva con respecto a la variedad de la muestra, los asistentes no rechazaron alimentos que pudieran no ser considerados de la zona, al contrario, este gusto se ha ido reconfigurando y entrelazando con platillos gastronómicos de otras zonas de México y el mundo.

Por su parte Infante (2008), se expresa con respecto a la resignificación performativa del cuerpo que en torno a los discursos gastronómicos los leemos en el contexto, los ciudadanos, lejos de sentirse ajenos a los nuevos alimentos que se van entretejiendo en el ambiente cultural culinario de la zona, se perfoman para darse la oportunidad de ampliar el gusto por nuevos horizontes gastronómicos sin salir de la isla.

Como se ha mencionado en el inicio del trabajo, Ciudad del Carmen, debido a su actividad económica y recursos naturales se ha erigido como un crisol cultural que ha ampliado la diversidad cultural culinaria de la isla, situación que ha enriquecido el capital cultural de sus habitantes.

De acuerdo con Borden (2002) que externa que las ciudades son sistemas complejos de representaciones, la realización de la Muestra Tradicional Carmelita manifiesta que cuando los ciudadanos están dispuestos a verbalizar el entorno a través de la comida, lejos de crearles conflicto sirve de escenario de confluencias para las narrativas culinarias, es decir, qué nos cuenta la comida, sus sabores, su chef, su cocinero, su repostero y cómo la cuenta quien la degusta.

\section{Conclusiones}

Con la creación de esta empresa cultural creativa se da fe que la metodología empresarial para detonar una mente emprendedora es cada vez más pertinente en los programas de estudio, este tipo de emprendimientos que demuestran en la práctica la creación de un Plan de Negocios, su implementación y su evaluación dotan de herramientas profesionales a los jóvenes universitarios.

Pero, aunado a lo anterior cuándo se habla de un emprendimiento empresarial de giro cultural se pudiera pensar que no es económicamente sostenible, los resultados de esta empresa demuestran que no es así, reafirma la importancia de la cultura como generadora de economía 
en las pequeñas comunidades en donde el consumo local es preponderante para la subsistencia de los pequeños y medianos emprendedores.

Cuando los ciudadanos ponen sobre la mesa el tema de la identidad cultural, los proyectos como el presentado en este trabajo de investigación demuestran que las empresas también pueden vender "cultura" a través de emprendimientos como el de la Muestra Tradicional Carmelita, que dicho de paso, es un proyecto inédito en la zona, pues hay algunos de temas específicos como los dedicados al camarón a través de festivales, pero, uno que reúna diversos rubros gastronómicos y que lo que prevalezca sea la degustación no lo hay hasta la presente fecha.

\section{Referencias}

Análisis del discurso: Una introducción (2ª edición). (2012). Bloomsbury Publishing. https://salahlibrary.files.wordpress.com/2018/09/discourse-analysis.pdf

Borden I. (2001) La ciudad desconocida: Arquitectura en disputa y espacio social: un proyecto extrañamente familiar. (2001). MIT Press (MA). https://doi.org/10.7551/mitpress/7008.001.0001

CANIRAC. (2019, 1 de enero). Todo sobre la mesa. Dimensiones de la Industria Restaurantera. Consultado el 4 de septiembre de 2021 en https://www.canirac.org.mx/images/publicidad/files/Todo\%20Sobre\%20la\%20Mesa.pdf

Hallensleben. (s.f.). Espacios Corporales Performativos: Topografías Corporales en Literatura, Teatro, Danza y Artes Visuales, Rodopi. Somatecnica, 1,434-437. https://www.euppublishing.com/toc/soma/1/2

Infante. (s.f.). Entrenando para ser transparentes. Buscando el cuerpo en el método de entrenamiento para actores propuesto por Jerzy Grotwski. Stichomythia,16-34. http://parnaseo.uv.es/Ars/Stichomythia/Stichomythia7/infante.pdf

Instituto Nacional de Estadística y Geografía (INEGI). (s.f.). Cultura. https://www.inegi.org.mx/temas/cultura/

Preciado. (s.f.). Cuerpo impropio. Guía de modelos somatopolíticos Y de sus posibles usos desviados. Resumen del seminario impartido POR Beatriz Preciado. https://paroledequeer.blogspot.com/. https://paroledequeer.blogspot.com/2014/11/cuerpo-impropioguia-de-modelos.html 